\title{
Control and Signaling in Distributed Linear Control Systems Allowing Choices*
}

\author{
Zhongchang Liu $^{1}$ and Wing Shing Wong ${ }^{1}$
}

\begin{abstract}
Distributed choice-based control systems are those where the controls exerted by the agents depend on the choices they have individually selected. They form an interesting class of decentralized systems in which control strategies are entangled with information structures. This paper focuses on linear systems that are jointly manipulated by multiple agents. We show how target-realizing controls depend on the information possessed by the agents and how control actions can serve as signaling tools. Related issues including feasibility and optimality of control laws are discussed.
\end{abstract}

\section{INTRODUCTION}

Decentralization of large-scale systems inevitably introduces non-classical information structure to distributed agents, i.e., agents cannot acquire the same information at the same time [1]. To operate as a group, communication becomes necessary and agents need to exchange with each other their individual observations, ideas or objectives. However, explicit communication systems (having physical communication channels, cable or wireless) may be costly in situations such as massive agents interaction or be forbidden and even unavailable to use in radio silence. Then, information may be disclosed by implicit signs. For example, people in a street may not be explicitly informed of a bad traffic congestion but may still deduce this fact by observing surrounding vehicles.

In a control system, the controlled plant can be regarded as a communication channel and control actions of each control agent, in addition to controlling the plant, can serve as a means for transmitting information. Implicit communication is thus informally defined in [2]. This idea has long been noticed since the celebrated "Witsenhausen's counterexample" [3]-[5]. Recently, there are increasing interests on direct application of controlled motion for information transmission [6] [7] and applied to interactive dance of human beings and robots [8]. Other examples of explicit communication are found in vehicle routing problems [9].

In this paper, we study the application of control to communicate choices of multiple agents. The choices can be interpreted as an agent's ideas, demands or objectives. For example, voting decisions in economic or political community, and set-points of domestic electrical appliances in a smart grid. Since the choice selected by an agent is only known to the agent itself, non-classical information

\footnotetext{
*This work is supported by the National Natural Science Foundation of China under grant number 61174060, and Shenzhen matching grant (GJHS 20120702105523301).

1 The authors are with Department of Information Engineering, The Chinese University of Hong Kong, Shatin, N.T., Hong Kong, and Shenzhen Research Institute, The Chinese University of Hong Kong. zcliulie.cuhk.edu.hk, wswong@ie.cuhk.edu.hk
}

structure is thus created in the system. Moreover, the system target is an amalgamated totality of the agents' choices. For illustration, consider a sensor positioning system where multiple users, each of whom has a bunch of areas to monitor, share a common sensor. Then, the target position of the sensor (defined as the circumcenter of all selected regions for example) will be determined by the selected areas of all agents. For all possible choice combinations, there will be a set of candidate targets. We are interested in investigating distributed algorithms that can realize any of these targets when chosen by the agents subject to such a information structure. This is called the choice-based target realization (CBTR) problem, which was first introduced in [10] [11] for information-based systems to determine the amount of information exchange and control cost.

This class of choice-based systems bring in new problems such as the feasibility of linear controllers and the increased cost incurred by the signaling role of controls. Without explicit communication, it is shown in [12] that the existence of CBTR control for a class of bilinear systems depends on the rank of the system mapping and the control cost is much higher than that with direct communication. For linear systems in [13], the feasibility of linear control laws is found to be determined by how the targets are organized and solutions for some constrained set of targets are derived.

In this paper, we further this line of investigation by concentrating on linear dynamical systems with a general set of targets. Control protocols are categorized by rounds of communication and control strategy cycles. Optimal control strategies of two control protocols are presented: the controllers of the single-round protocol are linear but can only solve CBTR problems with target sets satisfying certain conditions to be specified later; the two-round protocol uses control actions for information signaling in one round so that arbitrary collection of targets can be realized. The price of control-signaling is that the control laws are nonlinear.

The remaining part of this paper is organized as follows: Section II states the problem formulation. In Section III, conditions for the targets to be realized without communications are presented and the optimal single-round control law is derived. In Section IV, the new control-signaling strategy is proposed and its performance is evaluated. A simulation example validates our results. Conclusions and future works are provided in Section VI.

\section{PRoblem Formulation}

Consider a set of $L$ control agents with index set $\mathcal{I}=$ $\{1,2, \cdots, L\}$. At some initial time, each agent $l \in \mathcal{I}$ makes 
a choice $i_{l} \in \mathcal{C}_{l}$ from its own choice set $\mathcal{C}_{l}=\left\{1,2, \ldots, N_{l}\right\}$ with probability $p_{l}^{i_{l}}\left(\sum_{i_{l} \in \mathcal{C}_{l}} p_{l}^{i_{l}}=1, \forall l \in \mathcal{I}\right)$. Corresponding to that choice $i_{l}$, a control law $u_{l}^{i_{l}}(t)$ is provided to a linear system with dynamics described by

$$
\dot{x}_{\hat{i}}(t)=A x_{\hat{i}}(t)+\sum_{l=1}^{L} B_{l} u_{l}^{i_{l}}(t), \quad x_{\hat{i}}\left(t_{0}\right)=x_{0},
$$

where $\hat{i} \in\left\{\left(i_{1}, i_{2}, \ldots, i_{L}\right): i_{1} \in \mathcal{C}_{1}, \ldots, i_{L} \in \mathcal{C}_{L}\right\}$ is an instance of the choice outcome combination of all agents, $x_{\hat{i}}(t) \in \mathbb{R}^{n}$ is the state, $u_{l}^{i_{l}}(t) \in \mathbb{R}^{m_{l}}, A \in \mathbb{R}^{n \times n}$, and $B_{l} \in \mathbb{R}^{n \times m_{l}}$. Define $\mathcal{U}_{\hat{i}}(t)=\left\{u_{1}^{i_{1}}(t), u_{2}^{i_{2}}(t), \cdots, u_{L}^{i_{L}}(t)\right\}$ as the control profile when agents 1 to $L$ have the joint choice outcome $\hat{i}=i_{1}, i_{2}, \cdots, i_{L}$. And denote the set of agent $l$ 's controls corresponding to its choice set $\mathcal{C}_{l}$ at time $t$ by $\mathcal{U}_{l}(t)=\left\{u_{l}^{i_{l}}(t), i_{l} \in \mathcal{C}_{l}\right\}$. Thus $\mathbf{U}(t)=\left\{\mathcal{U}_{l}(t), l \in \mathcal{I}\right\}$ contains all control laws of all agents.

For any $\hat{i}$ there is a system output target, $H_{\hat{i}}$, to be reached at terminate time $t_{f}$. Then, there will be $N_{1} \cdot N_{2} \cdot \ldots \cdot N_{L}$ targets in total (some of them may have the same values), and they can be compactly represented and cited as entries of a target tensor, $\mathbf{H}=\left[H_{\hat{i}}\right]$. These targets represent different objectives of the system corresponding to the choice outcomes of the agents and are assumed pre-defined.

As a decentralized control system, each agent has no priori information about the others' choice decisions at the initial time, so the target state to be reached is undetermined to each agent and communication policies may be required in order to achieve it. Here arises the so called choice-based target realization (CBTR) problem: for a given target tensor $\mathbf{H}$, find control protocols (control and communication strategies) for the $L$ agents such that any target state $H_{\hat{i}}$ in $\mathbf{H}$, when chosen by the agents at the initial time $t_{0}$, can be realized within finite time $\left[t_{0}, t_{f}\right]$, i.e., make sure

$$
x_{\hat{i}}\left(t_{f}\right):=x\left(t_{f}, \mathcal{U}_{\hat{i}}\right)=H_{\hat{i}} .
$$

If a control protocol allows explicit communication among agents so that agents can fully disclose their choices to each other, then the problem reduces to a centralized one and the control strategy would be a series of target-reaching control sets $\left\{u_{1}^{\hat{i}}, u_{2}^{\hat{i}}, \cdots, u_{L}^{\hat{i}}\right\}$, one for each target $H_{\hat{i}}$. We call the control strategies in such a control protocol choicefree. However, without direct communication channels, an arbitrarily given target tensor $\mathbf{H}$ may not be realized (see section III). Hence, control actions may be used as means for information signaling.

Divide the time horizon $\left[t_{0}, t_{f}\right]$ into finite number of intervals $\left[t_{0}, t_{1}\right],\left[t_{1}, t_{2}\right], \cdots,\left[t_{k-1}, t_{f}\right]$ and call the interval $\left[t_{j-1}, t_{j}\right]$ as round $j$. In each round $\left[t_{j-1}, t_{j}\right]$, agents observe the system information at $t_{j-1}$ and then exert control actions, either for the purpose of signaling or for control, to the system. And the system runs in open-loop during each round. In the following sections, we design single-round control protocols in section III and two-round in section IV which shows how control actions are used for signaling.
In this paper, we also want to minimize the following averaged performance indicator

$$
\begin{aligned}
J(\mathbf{U}, \mathbf{H}, \gamma)= & \frac{1}{2} \int_{t_{0}}^{t_{f}} \sum_{l=1}^{L} \sum_{i_{l}=1}^{N_{l}} p_{l}^{i_{l}} u_{l}^{i_{l}}(t)^{T} u_{l}^{i_{l}}(t) d t \\
& +\frac{\gamma}{2} \sum_{\{\hat{i}\}} p_{\hat{i}}\left[x_{\hat{i}}\left(t_{f}\right)-H_{\hat{i}}\right]^{T}\left[x_{\hat{i}}\left(t_{f}\right)-H_{\hat{i}}\right],
\end{aligned}
$$

where $0 \leq \gamma \in \mathbb{R}$ is a weighting coefficient, and $p_{\hat{i}}=$ $\prod_{l=1}^{L} p_{l}^{i_{l}}$ is the joint probability of the choice outcome $\hat{i}$. This performance indicator concerns global optimality of each agent's control laws.

To simplify the analysis, we make the following assumption without loss of generality.

Assumption 1: Each item in the choice set of an agent has an equal probability to be chosen by that agent, i.e. for every $l \in \mathcal{I}, p_{l}^{i_{l}}=\frac{1}{N_{l}}, \forall i_{l} \in \mathcal{C}_{l}$.

So, the above cost function is rewritten as

$$
\begin{aligned}
& J(\mathbf{U}, \mathbf{H}, \gamma)=\frac{1}{2} \int_{t_{0}}^{t_{f}} \sum_{l=1}^{L} \frac{1}{N_{l}} \sum_{i_{l}=1}^{N_{l}} u_{l}^{i_{l}}(t)^{T} u_{l}^{i_{l}}(t) d t \\
& +\frac{\gamma}{2 \prod_{l=1}^{L} N_{l}} \sum_{\{\hat{i}\}}\left[x_{\hat{i}}\left(t_{f}\right)-H_{\hat{i}}\right]^{T}\left[x_{\hat{i}}\left(t_{f}\right)-H_{\hat{i}}\right]
\end{aligned}
$$

Assumption 2: System (1) is controllable by each agent, i.e., for $l=1,2, \ldots, L,\left(A, B_{l}\right)$ are controllable pairs, or the controllability Grammians

$$
W_{l}\left(t_{0}, t_{f}\right)=\int_{t_{0}}^{t_{f}} \Phi\left(t_{f}, \tau\right) B_{l} B_{l}^{T} \Phi^{T}\left(t_{f}, \tau\right) d \tau
$$

are invertible, where $\Phi(t, s)=e^{A(t-s)}$ is the transition matrix.

This assumption highlights that all agents share the same system information except each others' choices. For general decentralized control systems, their control problems with choices could be very difficult and remain open.

Notations: $I$ is an identity matrix of proper dimension. For brevity, we use the following notations: $\sum_{\mathcal{I}} \triangleq \sum_{k=1}^{L}$, $\sum_{\mathcal{I} / l} \triangleq \sum_{k=1, k \neq l}^{L}, \quad \sum_{\{\hat{i}\}} \triangleq \sum_{i_{1}=1}^{N_{1}} \sum_{i_{2}=1}^{N_{2}} \cdots \sum_{i_{L}=1}^{N_{L}}$, $\sum_{\mathcal{I} / l} \triangleq \sum_{\{\hat{i}\} /\left\{i_{l}\right\}} \triangleq \sum_{i_{1}=1}^{N_{1}} \cdots \sum_{i_{l-1}=1}^{N_{l-1}} \sum_{i_{l+1}=1}^{N_{l+1}} \cdots \sum_{i_{L}=1}^{N_{L}}=$ $\sum_{k \in \mathcal{I} / l} \sum_{i_{k}=1}^{N_{k}}$, and similar abbreviations apply to product $\prod$.

\section{Single-Round Control Protocols}

As introduced in Section II, single-round protocol is simply an open-loop control strategy. This section first characterizes the set of target tensors that are realizable by singleround protocols. Then, the optimal control laws minimizing the performance indicator (3) are derived.

\section{A. Feasibility of Single-Round Protocols}

The condition of existence of open-loop solutions can be stated in terms of structure conditions of the target tensor as discussed in [13]. For completeness of this paper, we briefly introduce its conclusion. 
It is well known that for system (1), a target state $H_{\hat{i}}$ can be realized at terminal time $t_{f}$ if and only if there exists a control profile $\mathcal{U}_{\hat{i}}(t)$ such that the following equation holds

$$
H_{\hat{i}}=\Phi\left(t_{f}, t_{0}\right) x_{\hat{i}}\left(t_{0}\right)+\int_{t_{0}}^{t_{f}} \Phi\left(t_{f}, t\right) \sum_{l=1}^{L} B_{l} u_{l}^{i_{l}}(t) d t .
$$

If it holds for all entries of a target tensor, one can verify by direct checking that for any indices, $i_{l}, i_{l}^{\prime} \in \mathcal{C}_{l}, i_{m}, i_{m}^{\prime} \in \mathcal{C}_{m}$, $l, m \in \mathcal{I}$, the following equality holds

$$
\begin{aligned}
H_{i_{1} \cdots i_{l} \cdots i_{m} \cdots i_{L}} & -H_{i_{1} \cdots i_{l} \cdots i_{m}^{\prime} \cdots i_{L}} \\
& =H_{i_{1} \cdots i_{l}^{\prime} \cdots i_{m} \cdots i_{L}}-H_{i_{1} \cdots i_{l}^{\prime} \cdots i_{m}^{\prime} \cdots i_{L}} .
\end{aligned}
$$

A target tensor $\mathbf{H}$ with all its entries satisfying condition (5) is called compatible, otherwise is called incompatible.

Theorem 1: [13] There exist single-round control protocols $\mathbf{U}(t)$ for linear system (1), such that $\mathbf{H}$ can be realized if and only if $\mathbf{H}$ is compatible.

Therefore, a given target tensor may be not realizable without communication strategies. For compatible target tensors, the optimal control laws minimizing a quadratic control cost function (14) exist and have been derived in [13] as well as in the next subsection in a more explicit form. For incompatible target tensors, signaling strategies should be added to result in a multi-round protocol as in Section IV-B

\section{B. Optimal Single-Round Controls}

Given the utility function (3), we can find optimal openloop controls and optimal terminal states $\hat{\mathbf{X}}\left(t_{f}\right)=\left\{\hat{x}_{\hat{i}}\left(t_{f}\right) \in\right.$ $\left.\mathbb{R}^{n}, \hat{i} \in \mathcal{C}_{1} \times \cdots \times \mathcal{C}_{L}\right\}$ to achieve its minimum.

Proposition 1: For an $n$-dimensional system with $L$ agents in (1), the set of single-round controls minimizing (3) is given by: $\forall l \in \mathcal{I}, i_{l} \in \mathcal{C}_{l}$,

$$
\hat{u}_{l}^{i_{l}}(t)=B_{l}^{T} \Phi^{T}\left(t_{f}, t\right) D_{l}^{i_{l}}\left(t_{f}, \gamma\right),
$$

the optimal terminal states at time $t_{f}$ are:

$$
\hat{x}_{\hat{i}}\left(t_{f}, \gamma\right)=\Phi\left(t_{f}, t_{0}\right) x_{0}+\sum_{l \in \mathcal{I}} W_{l}\left(t_{0}, t_{f}\right) D_{l}^{i_{l}}\left(t_{f}, \gamma\right),
$$

and the optimal cost is:

$$
\begin{aligned}
J_{o}^{*}(\mathbf{H}, \gamma)= & \frac{1}{2 \prod_{\mathcal{I}} N_{k}} \sum_{\{\hat{i}\}}\left[\gamma e_{\hat{i}}^{T}\left(t_{f}, \gamma\right) e_{\hat{i}}\left(t_{f}, \gamma\right)\right. \\
& \left.+\sum_{l \in \mathcal{I}} D_{l}^{i_{l}}\left(t_{f}, \gamma\right)^{T} W_{l}\left(t_{0}, t_{f}\right) D_{l}^{i_{l}}\left(t_{f}, \gamma\right)\right]
\end{aligned}
$$

where

$$
\begin{gathered}
D_{l}^{i_{l}}\left(t_{f}, \gamma\right)=\gamma\left(I+\gamma \sum_{\mathcal{I}} W_{k}\right)^{-1} \frac{\sum_{\{\hat{i}\}} d_{\hat{i}}\left(t_{0}, t_{f}\right)}{\prod_{\mathcal{I}} N_{k}}+ \\
\gamma\left(I+\gamma W_{l}\right)^{-1}\left(\frac{\sum_{\{\hat{i}\} \backslash\left\{i_{l}\right\}} d_{\hat{i}}\left(t_{0}, t_{f}\right)}{\prod_{\mathcal{I} \backslash l} N_{k}}-\frac{\sum_{\{\hat{i}\}} d_{\hat{i}}\left(t_{0}, t_{f}\right)}{\prod_{\mathcal{I}} N_{k}}\right) \\
d_{\hat{i}}\left(t_{0}, t_{f}\right)=H_{\hat{i}}-\Phi\left(t_{f}, t_{0}\right) x_{0}
\end{gathered}
$$

and

$$
e_{\hat{i}}\left(t_{f}, \gamma\right)=H_{\hat{i}}-\hat{x}_{\hat{i}}\left(t_{f}, \gamma\right)
$$

$$
=d_{\hat{i}}\left(t_{0}, t_{f}\right)-\sum_{l \in \mathcal{I}} W_{l}\left(t_{0}, t_{f}\right) D_{l}^{i_{l}}\left(t_{f}, \gamma\right)
$$

The proof is omitted owing to space limitation.

Note that the above controls minimize the cost function (3) but may be unable to solve the CBTR problem. To see this, let $\gamma \rightarrow \infty$, and then the terminal state

$$
\begin{aligned}
& \hat{x}_{\hat{i}}\left(t_{f}, \infty\right)=\Phi\left(t_{f}, t_{0}\right) x_{0}+\sum_{l \in \mathcal{I}} W_{l} D_{l}^{i_{l}}\left(t_{f}, \infty\right) \\
& =\Phi\left(t_{f}, t_{0}\right) x_{0}+\sum_{l \in \mathcal{I}}\left[W_{l}\left(\sum_{\mathcal{I}} W_{k}\right)^{-1} \frac{\sum_{\{\hat{i}\}} d_{\hat{i}}}{\prod_{\mathcal{I}} N_{k}}\right. \\
& \left.+\left(\frac{\sum_{\{\hat{i}\} \backslash\left\{i_{l}\right\}} d_{\hat{i}}}{\prod_{\mathcal{I} \backslash l} N_{k}}-\frac{\sum_{\{\hat{i}\}} d_{\hat{i}}}{\prod_{\mathcal{I}} N_{k}}\right)\right] \\
& =\Phi\left(t_{f}, t_{0}\right) x_{0}+\sum_{l \in \mathcal{I}}\left(\frac{\sum_{\{\hat{i}\} \backslash\left\{i_{l}\right\}} d_{\hat{i}}}{\prod_{\mathcal{I} \backslash l} N_{k}}\right)-\frac{(L-1) \sum_{\{\hat{i}\}} d_{\hat{i}}}{\prod_{\mathcal{I}} N_{k}} \\
& =\sum_{l \in \mathcal{I}}\left(\frac{\sum_{\{\hat{i}\} \backslash\left\{i_{l}\right\}} H_{\hat{i}}}{\prod_{\mathcal{I} \backslash l} N_{k}}\right)-\frac{(L-1) \sum_{\{\hat{i}\}} H_{\hat{i}}}{\prod_{\mathcal{I}} N_{k}}
\end{aligned}
$$

is, in general, not equal to the target $H_{\hat{i}}$, i.e., $\exists \hat{i}, e_{\hat{i}}\left(t_{f}, \infty\right) \neq$ 0 . This proves in a different manner from Theorem 1 that the realizability of a target tensor (i.e. whether all entries of $\mathbf{H}$ can be realized) cannot be guaranteed by single round protocols. But, for target tensors that are compatible, the CBTR controls can be readily obtained following from Proposition 1.

Theorem 2: For an $n$-dimensional system with $L$ agents in (1), the following set of controls: $\forall l \in \mathcal{I}, i_{l} \in \mathcal{C}_{l}$,

$$
\hat{u}_{l}^{i_{l}}(t)=B_{l}^{T} \Phi^{T}\left(t_{f}, t\right) D_{l}^{i_{l}}\left(t_{f}, \infty\right)
$$

can realize a compatible target tensor $\mathbf{H}$ while minimize the control cost function

$$
J_{s}(\mathbf{U})=\frac{1}{2} \int_{t_{0}}^{t_{f}} \sum_{l \in \mathcal{I}} \frac{1}{N_{l}} \sum_{i_{l}=1}^{N_{l}}\left(u_{l}^{i_{l}}(t)\right)^{T} u_{l}^{i_{l}}(t) d t .
$$

Proof: Since the controls in this theorem follows directly from Proposition 1, we only need to show that all entries of a compatible $\mathbf{H}$ can be exactly reached. First, recall from [13] that every entry of a compatible $\mathbf{H}$ can be expressed by

$H_{i_{1} i_{2} \cdots i_{L}}=H_{i_{1} 1 \cdots 1}+H_{1 i_{2} 1 \cdots 1}+\cdots H_{1 \cdots 1 i_{L}}-(L-1) H_{1 \cdots 1}$.

Then, solving the system equation with the above control inputs, one will get the same solutions as in (12) for every terminal state $x_{\hat{i}}\left(t_{f}\right)$. Now, considering an arbitrary choice event of all agents $i_{1}^{*} i_{2}^{*} \cdots i_{L}^{*}$, the corresponding terminal state will be

$$
\begin{aligned}
& x_{i_{1}^{*} i_{2}^{*} \cdots i_{L}^{*}}\left(t_{f}\right)=\sum_{l \in \mathcal{I}}\left(\frac{\sum_{\{\hat{i}\} /\left\{i_{l}^{*}\right\}} H_{\hat{i}}}{\prod_{\mathcal{I} / l} N_{k}}\right)-(L-1) \frac{\sum_{\{\hat{i}\}} H_{\hat{i}}}{\prod_{\mathcal{I}} N_{k}} \\
& =\frac{1}{\prod_{\mathcal{I}} N_{k}}\left[\left(N_{1} \sum_{\{\hat{i}\} /\left\{i_{1}^{*}\right\}} H_{\hat{i}}-\sum_{\{\hat{i}\}} H_{\hat{i}}\right)+\left(N_{2} \sum_{\{\hat{i}\} /\left\{i_{2}^{*}\right\}} H_{\hat{i}}\right.\right. \\
& \left.\left.-\sum_{\{\hat{i}\}} H_{\hat{i}}\right)+\cdots+\left(N_{L} \sum_{\{\hat{i}\} /\left\{i_{L}^{*}\right\}} H_{\hat{i}}-\sum_{\{\hat{i}\}} H_{\hat{i}}\right)+\sum_{\{\hat{i}\}} H_{\hat{i}}\right]
\end{aligned}
$$




$$
\begin{aligned}
&=\frac{1}{\prod_{\mathcal{I}} N_{k}} {\left[\prod_{\mathcal{I} / 1} N_{k}\left(N_{1} H_{i_{1}^{*} 1 \cdots 1}-\sum_{i_{1}=1}^{N_{1}} H_{i_{1} 1 \cdots 1}\right)\right.} \\
&+\prod_{\mathcal{I} / 2} N_{k}\left(N_{2} H_{1 i_{2}^{*} 1 \cdots 1}-\sum_{i_{2}=1}^{N_{2}} H_{1 i_{2} 1 \cdots 1}\right)+\cdots \\
&+\prod_{\mathcal{I} / L} N_{k}\left(N_{L} H_{1 \cdots 1}-\sum_{i_{L}=1}^{N_{L}} H_{1 \cdots 1 i_{L}}\right) \\
&+\left(\prod_{\mathcal{I} / 1} N_{k} \sum_{i_{1}=1}^{N_{1}} H_{i_{1} 1 \cdots 1}+\prod_{\mathcal{I} / 2} N_{k} \sum_{i_{2}=1}^{N_{2}} H_{1 i_{2} 1 \cdots 1}+\cdots\right. \\
&\left.\left.+\prod_{\mathcal{I} / L} N_{k} \sum_{i_{L}=1}^{N_{L}} H_{1 \cdots 1 i_{L}}-\prod_{\mathcal{I}} N_{k}(L-1) H_{1 \cdots 1}\right)\right] \\
&\left.=H_{i_{1}^{*} 1 \cdots 1}+H_{1 i_{2}^{*} 1 \cdots 1}+\cdots+H_{1 \cdots 1}\right) \\
&=H_{i_{1}^{*} i_{2}^{*} \cdots i_{L}^{*}} .
\end{aligned}
$$

This completes the proof.

Remark 1: In [13], equivalent optimal solutions for compatible target tensors have been derived, but each control profile $\mathcal{U}_{\hat{i}}$ needs to be computed as a whole. In contrast, each control input in (13) has a separate expression, which reduces the computational complexity for each agent.

\section{Multi-Round Control Protocol}

In this section, we propose multi-round protocols which can solve the CBTR problem for arbitrarily defined target tensors. Before that, the choice-free control law is presented first, which can serve in a basic step of the multi-round protocol and the induced cost can be considered as a benchmark for control protocols with signaling strategies.

\section{A. Optimal choice-free control strategy}

If the agents' choices are fully disclosed to each other in advance using any communication strategy, we get a series of classical choice-free control problems whose optimal solutions can be derived by minimizing a sequence of utility functions: $\forall \hat{i} \in \mathcal{C}_{1} \times \cdots \times \mathcal{C}_{L}$,

$$
\begin{aligned}
J_{\hat{i}}\left(\mathcal{U}_{\hat{i}}, H_{\hat{i}}, \gamma\right)= & \frac{1}{2} \int_{t_{0}}^{t_{f}} \sum_{l \in \mathcal{I}}\left(u_{l}^{i_{l}}(t)\right)^{T} u_{l}^{i_{l}}(t) d t \\
& +\frac{\gamma}{2}\left[x_{\hat{i}}\left(t_{f}\right)-H_{\hat{i}}\right]^{T}\left[x_{\hat{i}}\left(t_{f}\right)-H_{\hat{i}}\right] .
\end{aligned}
$$

By standard procedures [14], the set of choice-free optimal controls realizing one target $H_{\hat{i}}$ is: for $l=1,2, \cdots, L$,

$$
\hat{u}_{l}^{\hat{i}}(t)=B_{l}^{T} \Phi^{T}\left(t_{f}, t\right) P_{\hat{i}}\left(t_{0}, \gamma\right),
$$

with

$$
P_{\hat{i}}\left(t_{0}, \gamma\right)=\gamma\left[I+\gamma \sum_{\mathcal{I}} W_{k}\left(t_{0}, t_{f}\right)\right]^{-1} d_{\hat{i}}\left(t_{0}, t_{f}\right),
$$

and each control of an agent relies explicitly on the other agents' choices. The optimal terminal state is

$$
\hat{x}_{\hat{i}}^{c}\left(t_{f}, \gamma\right)=H_{\hat{i}}-\frac{1}{\gamma} P_{\hat{i}}\left(t_{0}, \gamma\right) .
$$

So, the optimal cost for a single target is derived as

$$
J_{\hat{i}}\left(\hat{\mathcal{U}}_{\hat{i}}, H_{\hat{i}}, \gamma\right)=\frac{1}{2 \gamma} P_{\hat{i}}^{T}\left(t_{0}, \gamma\right)\left(I+\gamma \sum_{\mathcal{I}} W_{k}\left(t_{0}, t_{f}\right)\right) P_{\hat{i}}\left(t_{0}, \gamma\right)
$$

and the averaged optimal choice-free cost is

$$
\begin{aligned}
& J_{c}^{*}(\mathbf{H}, \gamma):=\frac{1}{\Pi_{\mathcal{I}} N_{k}} \sum_{\{\hat{i}\}} J_{\hat{i}}\left(\hat{\mathcal{U}}_{\hat{i}}, H_{\hat{i}}, \gamma\right) \\
& =\frac{1}{2 \gamma \Pi_{\mathcal{I}} N_{k}} \sum_{\{\hat{i}\}} P_{\hat{i}}^{T}\left(t_{0}, \gamma\right)\left(I+\gamma \sum_{\mathcal{I}} W_{k}\left(t_{0}, t_{f}\right)\right) P_{\hat{i}}\left(t_{0}, \gamma\right) .
\end{aligned}
$$

Letting $\gamma \rightarrow \infty$, (18) shows that $\hat{x}_{\hat{i}}^{c}\left(t_{f}, \infty\right)=H_{\hat{i}}$. Hence, the CBTR problem can be solved for arbitrary target tensors if a communication strategy can be introduced into control protocols. But, this protocol requires auxiliary communication devices and channels. In the following, we will introduce a two-round control protocol, the control-signaling strategy, which does not rely on additional communication resources.

\section{B. Control-Signaling Strategy}

There have been various attempts of designing signaling strategies as discussed in the Introduction. The basic idea is to identify information from distinguishable system outputs. Since we don't consider disturbances in systems, control protocols containing two rounds can be used: in the first round (signaling round), agents use their control inputs to indicate their choices via the system; in the second round (control round), agents make observations, map the observed system output to the corresponding target and apply controls to achieve that desired target. We call such a protocol a Control Signaling Strategy (CSS).

In the following, we propose a code-tensor approach to indicate choices in the signaling round. Concretely, the target tensor $\mathbf{H}$ is encoded to another tensor, named code tensor. For $\tilde{\mathbf{H}}=\left[\tilde{H}_{\hat{i}}\right]$ to be a code tensor for a target tensor $\mathbf{H}$, it should satisfy the following conditions

1) $\tilde{\mathbf{H}}$ has the same dimension as $\mathbf{H}$,

2) all entries $\tilde{H}_{\hat{i}}$ of $\tilde{\mathbf{H}}$ are achievable by system (1) without information signaling; this is equivalent to say that $\tilde{\mathbf{H}}$ should be compatible,

3) and every $\tilde{H}_{\hat{i}}$ can be distinguished from each other by each agent.

Then, in the signaling round, from time $t_{0}$ to some agreed intermediate time $t_{1}<t_{f}$, agents steer the system state to reach an entry of the code tensor by applying choice-based control law $u_{l}^{i_{l}}(t) \in \psi_{s}\left(x_{0}, \tilde{\mathbf{H}}\right):=\left\{\psi_{s}^{i_{l}}\left(x_{0}, \tilde{\mathbf{H}}\right), i_{l} \in \mathcal{C}_{l}, l \in\right.$ $\mathcal{I}$ \}. At time $t_{1}$, every agent decides a target state from $\mathbf{H}$ by decoding the observed system state $x\left(t_{1}\right)$ independently. Finally, every agents applies their own choice-free control laws $u_{l}^{\hat{i}}(t) \in \psi_{c}\left(\mathbf{X}\left(t_{1}\right), \mathbf{H}\right):=\left\{\psi_{c, l}\left(x_{\hat{i}}\left(t_{1}\right), H_{\hat{i}}\right), l \in \mathcal{I}, \hat{i} \in\right.$ $\left.\mathcal{C}_{1} \times \cdots \times \mathcal{C}_{L}\right\}$ to steer the system towards the decided target during the time horizon $\left[t_{1}, t_{f}\right]$ of the control round.

Remark 2: The critical part of this CSS is reliable information decoding from the observed output at $t_{1}$. For deterministic systems, one signaling round with very low 
control cost is adequate to indicate without error the selected target. For systems with disturbances, like a noisy communication channel, more rounds of control-signaling might be applied so as to guarantee reliable information transmission. In [15], the authors considered a choice inference strategy for stochastic systems which allows two agents to infer each other's choice with multiple rounds of observations. But that inferring strategy is for compatible target tensors only and the complexity grows fast as the number of agents increases.

Given $x_{0}, \mathbf{H}$ and a designed code tensor $\tilde{\mathbf{H}}$, suppose $\left(\psi_{s}^{*}\left(x_{0}, \tilde{\mathbf{H}}\right), \psi_{c}^{*}(\tilde{\mathbf{H}}, \mathbf{H})\right)$ is the set of optimal CSS for system (1) subject to the utility function (3). Then,

$$
\begin{aligned}
J_{c s}(\mathbf{U}, \tilde{\mathbf{H}}, \mathbf{H}, \gamma):=J\left(\left(\psi_{s}, \psi_{c}\right), \mathbf{H}, \gamma\right) \\
=\frac{1}{2} \int_{t_{0}}^{t_{1}} \sum_{l \in \mathcal{I}} \frac{1}{N_{l}} \sum_{i_{l}=1}^{N_{l}}\left(u_{l}^{i_{l}}(t)\right)^{T} u_{l}^{i_{l}}(t) d t \\
\quad+\frac{1}{2} \int_{t_{1}}^{t_{f}} \sum_{l \in \mathcal{I}} \frac{1}{N_{l}} \sum_{i_{l}=1}^{N_{l}}\left(u_{l}^{i_{l}}(t)\right)^{T} u_{l}^{i_{l}}(t) d t \\
\quad+\frac{\gamma}{2 \prod_{l \in \mathcal{I}} N_{l}} \sum_{\{\hat{i}\}}\left[x_{\hat{i}}\left(t_{f}\right)-H_{\hat{i}}\right]^{T}\left[x_{\hat{i}}\left(t_{f}\right)-H_{\hat{i}}\right] \\
=J_{s}\left(\psi_{s}\left(x_{0}, \tilde{\mathbf{H}}\right)\right)+J_{c}\left(\psi_{c}(\tilde{\mathbf{H}}, \mathbf{H}), \gamma\right) \\
\geq J_{s}\left(\psi_{s}^{*}\left(x_{0}, \tilde{\mathbf{H}}\right)\right)+J_{c}\left(\psi_{c}^{*}(\tilde{\mathbf{H}}, \mathbf{H}), \gamma\right)=: J_{c s}^{*}(\tilde{\mathbf{H}}, \mathbf{H}, \gamma)
\end{aligned}
$$

where $J_{s}$ and $J_{c}$ are defined in (14) and (19), respectively. The optimal cost $J_{c s}^{*}(\tilde{\mathbf{H}}, \mathbf{H}, \gamma)$ subject to $\tilde{\mathbf{H}}$ is achieved by the following control law: during the signaling round (cf. (13))

$$
\hat{u}_{l}^{i_{l}}(t)=B_{l}^{T} \Phi^{T}\left(t_{1}, t\right) D_{l}^{i_{l}}\left(t_{1}, \infty\right), t_{0} \leq t \leq t_{1},
$$

while during the control round (cf. (16))

$$
\hat{u}_{l}^{\hat{i}}(t)=B_{l}^{T} \Phi^{T}\left(t_{f}, t\right) P_{\hat{i}}\left(t_{1}, \gamma\right), t_{1} \leq t \leq t_{f}
$$

where $d_{\hat{i}}\left(t_{0}, t_{1}\right)=\tilde{H}_{\hat{i}}-\Phi\left(t_{1}, t_{0}\right) x_{0}$ in $D_{l}^{i_{l}}\left(t_{1}, \infty\right)$, and $d_{\hat{i}}\left(t_{1}, t_{f}\right)=H_{\hat{i}}-\Phi\left(t_{f}, t_{1}\right) \tilde{H}_{\hat{i}}$ in $P_{\hat{i}}\left(t_{1}, \gamma\right)$.

Remark 3: Notice that the controllers of the CSS are not linear any more. The loss of linear structure of optimal controllers for decentralized linear systems has long been noted when non-classical information structures were encountered originally [1]. It was conjectured before that nothing was to be gained by using nonlinear controllers when the objective is to minimize a quadratic criterion for a linear system with unconstrained control variables. For the stochastic Witsenhausen's counterexample [3], [16], the optimal controller, although is still unknown [5], is not linear. Nonlinear signaling control strategies can outperform linear control strategies by an arbitrarily large ratio [4]. In the following we draw the same conclusion for linear deterministic control systems with distributed choice actions.

\section{Cost of the Control-Signaling Strategy}

To compute the system cost with a CSS, we define code tensors as follows.

Defination 1: Given $\delta>0$, if all entries of a finite dimensional tensor $\mathbf{T}=\left[T_{\hat{i}} \in \mathbb{R}^{n}\right]$, which has the same dimensions as a target tensor $\mathbf{H}$, satisfy (5) and the Euclidean distance $\left\|T_{\hat{i}}-T_{\hat{i}^{\prime}}\right\| \geq \delta, \forall \hat{i} \neq \hat{i}^{\prime}, \hat{i}, \hat{i}^{\prime} \in \mathcal{C}_{1} \times \cdots \times \mathcal{C}_{L}$, then $\mathbf{T}$ can be a code tensor for $\mathbf{H}$.

The optimal code tensor which can minimize the utility function (3) should be the minimizer of the following quadratic programming problem:

$$
\begin{array}{cl}
\min _{\mathbf{T}} & J_{c s}^{*}(\mathbf{T}, \mathbf{H}, \gamma) \\
\text { s. t. } & \left\|T_{\hat{i}}-T_{\hat{i}^{\prime}}\right\| \geq \delta, \quad \forall \hat{i} \neq \hat{i}^{\prime} \\
& T_{\hat{i}} \in \mathbb{R}^{n}, \quad \forall \hat{i}, \hat{i}^{\prime} \in \mathcal{C}_{1} \times \cdots \times \mathcal{C}_{L} .
\end{array}
$$

However, this optimization problem is not convex and may be hard to solve. In the following, we design code tensors which have simple lattice structures.

Let $\Delta \in \mathbb{R}^{n}$ be a vector with Euclidean length $\|\Delta\|=\delta$. Let

$$
\tilde{H}_{\hat{i}}=s_{\hat{i}} \Delta+\Phi\left(t_{1}, t_{0}\right) x_{0}
$$

where

$$
\begin{aligned}
s_{\hat{i}}= & \left(i_{1}-1\right) N_{2} \cdots N_{L}+\left(i_{2}-1\right) N_{3} \cdots N_{L} \\
& +\cdots+\left(i_{L-1}-1\right) N_{L}+\left(i_{L}-1\right) \\
= & \sum_{l \in \mathcal{I}}\left(i_{l}-1\right) \prod_{k=l+1}^{L} N_{k}
\end{aligned}
$$

is a bijection from $\mathcal{C}_{1} \times \cdots \times \mathcal{C}_{L}$ to $\left\{0,1, \ldots, N_{1} N_{2} \cdots N_{L}\right\}$. Since $s_{i_{1} \cdots i_{l} \cdots i_{m} \cdots i_{L}}-s_{i_{1} \cdots i_{l} \cdots i_{m}^{\prime} \cdots i_{L}}=\left(i_{m}-i_{m}^{\prime}\right) \times N_{m+1} \times$ $\cdots \times N_{L}=s_{i_{1} \cdots i_{l}^{\prime} \cdots i_{m} \cdots i_{L}}-s_{i_{1} \cdots i_{l}^{\prime} \cdots i_{m}^{\prime} \cdots i_{L}}$, and $\forall \hat{i} \neq \hat{i}^{\prime}$, $\left\|\tilde{H}_{\hat{i}}-\tilde{H}_{\hat{i}^{\prime}}\right\|=\|k \Delta\|=|k| \delta, k \in \mathbb{Z} \backslash\{0\}$, the tensor $\left[\tilde{H}_{\hat{i}}\right]$ thus defined can be a code tensor for $\mathbf{H}$.

Then the nonlinear control law in (20) (21) with (22) can outperform the linear control law (6) as stated below.

Theorem 3: For system (1) with an incompatible target tensor $\mathbf{H}$ and a corresponding code tensor $\tilde{\mathbf{H}}$, there exists a finite $\gamma_{1}>0$, such that $J_{c s}^{*}(\tilde{\mathbf{H}}, \gamma)<J_{o}^{*}(\mathbf{H}, \gamma)$ whenever $\gamma>\gamma_{1}$.

So, by using control for signaling, the control laws sacrifice the linear structure but accomplish tasks that linear control strategies cannot and may incur less cost compared with linear strategies.

We can also prove that both costs induced by the CSS and that of single-round protocols are always larger than that by the choice-free strategy which uses explicit communication. A numerical result in section $\mathrm{V}$ also validates this point. So, this increased portion of cost may be considered as the price of communication.

\section{AN EXAMPLE}

Consider the sensor positioning system with three agents $\mathbf{a}, \mathbf{b}$ and $\mathbf{c}$, each of whom has two regions marked in $\mathrm{X}-\mathrm{Y}$ coordinates in Fig. 1. The motion of the sensor is described by the C-W equation (1) with $x=\left[x_{1}, x_{2}, x_{3}, x_{4}\right]^{T}$ and system parameters [17]

$$
A=\left[\begin{array}{cccc}
0 & 1 & 0 & 0 \\
0 & 0 & 0 & 2 \omega \\
0 & 0 & 0 & 1 \\
0 & -2 \omega & 3 \omega^{2} & 0
\end{array}\right], B_{l}=\left[\begin{array}{ll}
0 & 0 \\
1 & 0 \\
0 & 0 \\
0 & 1
\end{array}\right], l=1,2,3
$$


where $x_{1}$ is the $\mathrm{X}$-coordinate position, $x_{3}$ is the Y-coordinate position and $\omega=7.2722 \times 10^{-5}$ radians/s is the sensor's orbit angular velocity.

The target positions are defined as the centers of the smallest circles containing the agents' selected regions. Thus, they are incompatible. The CSS in (20) (21) with code tensor (22) was applied to generate the trajectories in Fig. 1, which shows that every target position can be realized if chosen by the agents. Fig. 2 shows that costs induced by the CSS $\left(J_{c s}^{*}\right)$ and by the choice-free strategy $(16)\left(J_{c}^{*}\right)$ are finite as $\gamma$ increases. But the cost with single round protocol $\left(J_{o}^{*}\right)$ blows up. This proves that single round protocols are infeasible for incompatible targets.

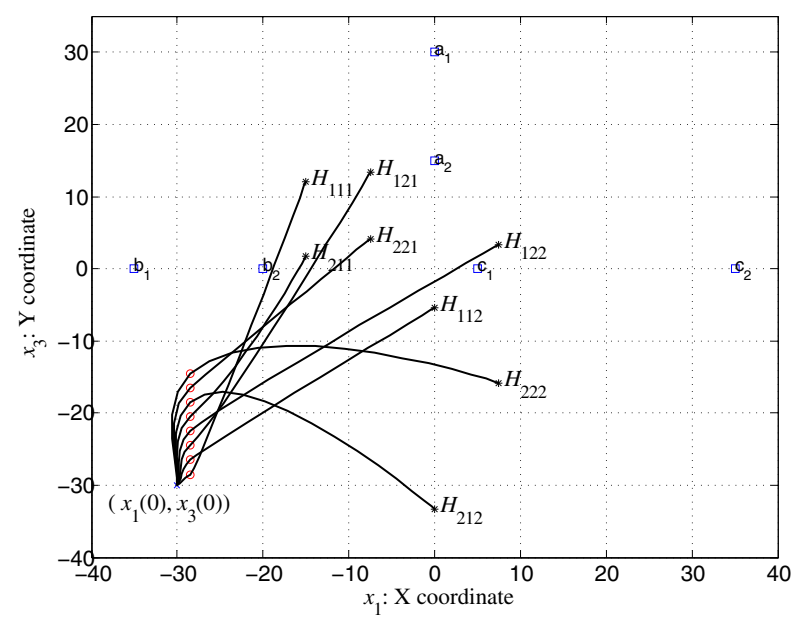

Fig. 1. All possible trajectories of the sensor according to the three agents' choices. $\square$ : coordinates of the agents' regions; $*$ : target positions of the sensor; ०: code positions.

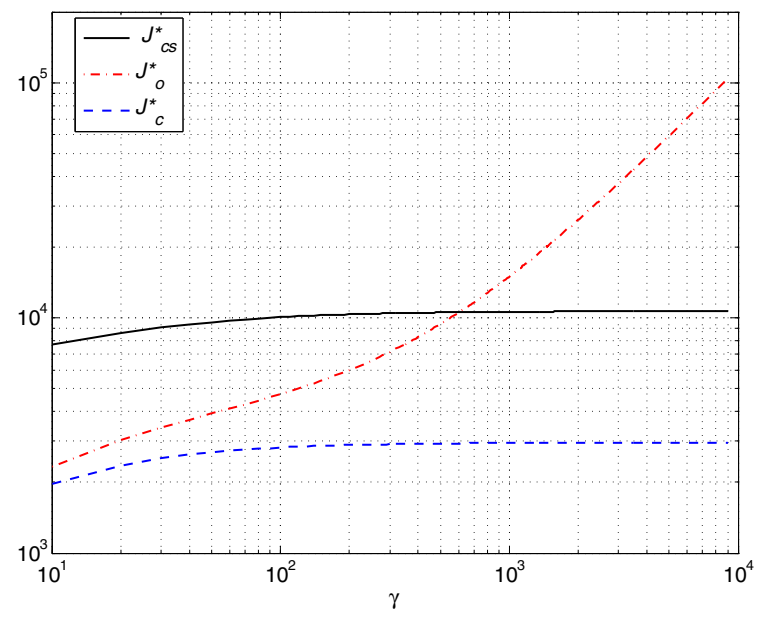

Fig. 2. Cost values with three control strategies vs $\gamma$. The CSS outperforms the optimal single-round control when $\gamma$ is larger than about 600 .

\section{CONCLUSIONS}

In the present paper we have investigated the control protocol design problem for linear choice-based action systems. Three protocols are proposed to solve the CBTR problem and optimal solutions are derived by minimizing an averaged cost function. Especially, a two-round control protocol is shown to be able to deal with incompatible target tensors. In the future, the performance of the CSS comparing with choicefree strategies should be analyzed so as to explore the value of communication bits. Also, design of control protocols for systems with disturbances, or observation noises is of great interest.

\section{REFERENCES}

[1] Y.-C. Ho, "Team decision theory and information structures," Proc. IEEE, vol. 68, no. 6, pp. 644-654, 1980.

[2] P. Grover, "Actions can speak more clearly than words," Ph.D. dissertation, EECS Department, University of California, Berkeley, Jan 2011. [Online]. Available: http://www. eecs.berkeley.edu/ Pubs/TechRpts/2011/EECS-2011-1.html

[3] H. S. Witsenhausen, "A counterexample in stochastic optimum control," SIAM J. Contr., vol. 6, no. 1, pp. 131-147, 1968.

[4] S. Mitter and A. Sahai, "Information and control: Witsenhausen revisited," in Learning, Control and Hybrid Systems: Lecture Notes in Control and Information Sciences 241, Y. Yamamoto and S. Hara, Eds. New York: Springer, 1999, pp. 281-293.

[5] P. Grover, S. Y. Park, and A. Sahai, "Approximately optimal solutions to the finite-dimensional witsenhausen counterexample," IEEE Trans. Autom. Control, vol. 58, no. 9, pp. 2189-2204, 2013.

[6] D. V. Raghunathan and J. Baillieul, "Exploiting information content in relative motion," in Proc. American Control Conference, St. Louis, USA, Dec.10-12, 2009, pp. 2166-2171.

[7] A. Jones and S. Andersson, "A motion-based communication system," in Proc. American Control Conference, Washington DC, USA, Jun.1719, 2013, pp. 365-370.

[8] J. Baillieul and K. Ozcimder, "The control theory of motion-based communication: Problems in teaching robots to dance," in Proc. American Control Conference, Montréal, Canada, Jun. 2012, pp. 43194326.

[9] A. Arsie, K. Savla, and E. Frazzoli, "Efficient routing algorithms for multiple vehicles with no explicit communications," IEEE Trans. Autom. Control, vol. 54, no. 10, pp. 2302-2317, Oct. 2009.

[10] W. S. Wong, "Control communication complexity of distributed control systems," SIAM J. Control Optim., vol. 48, no. 3, pp. 1722-1742, May 2009.

[11] W. S. Wong and J. Baillieul, "Control communication complexity of nonlinear systems," Communications in Information and Systems, vol. 9, no. 1, pp. 103-140, May 2009.

[12] - "Control communication complexity of distributed actions," IEEE Trans. Autom. Control, vol. 57, no. 11, pp. 2731-2745, Nov. 2012.

[13] G. Guo, Z. Liu, and W. S. Wong, "Joint target realization for linear systems allowing choice-based actions," submitted, 2013. [Online]. Available: http://www.ie.cuhk.edu.hk/people/documents/ submit.pdf

[14] A. E. Bryson and Y.-C. Ho, Applied optimal control: optimization, estimation, and control. New York: Taylor \& Francis, 1975.

[15] Z. Liu, W. S. Wong, and G. Guo, "Cooperative control of linear systems with choice actions," in Proc. American Control Conference, Washington DC, USA, Jun.17-19, 2013, pp. 5394-5399.

[16] R. Bansal and T. Başar, "Stochastic teams with nonclassical information revisited: When is an affine law optimal?" IEEE Trans. Autom. Control, vol. 32, no. 6, pp. 554-559, 1987.

[17] V. Kapila, A. G. Sparks, J. M. Buffington, and Q. Yan, "Spacecraft formation flying: Dynamics and control," Journal of Guidance, Control, and Dynamics, vol. 23, no. 3, pp. 561-564, 2000. 